\title{
Thickly Syndetical Sensitivity of Topological Dynamical System
}

\author{
Heng Liu, ${ }^{1,2}$ Li Liao, ${ }^{3}$ and Lidong Wang ${ }^{2}$ \\ ${ }^{1}$ Department of Mathematics, Dalian University of Technology, Dalian, Liaoning 116024, China \\ ${ }^{2}$ Department of Mathematics, Dalian Nationalities University, Dalian, Liaoning 116600, China \\ ${ }^{3}$ Institute of Applied Physics and Computational Mathematics, Beijing 100094, China
}

Correspondence should be addressed to Heng Liu; hliu@dlnu.edu.cn

Received 7 January 2014; Accepted 1 April 2014; Published 24 April 2014

Academic Editor: Mingshu Peng

Copyright (C) 2014 Heng Liu et al. This is an open access article distributed under the Creative Commons Attribution License, which permits unrestricted use, distribution, and reproduction in any medium, provided the original work is properly cited.

Consider the surjective continuous map $f: X \rightarrow X$, where $X$ is a compact metric space. In this paper we give several stronger versions of sensitivity, such as thick sensitivity, syndetic sensitivity, thickly syndetic sensitivity, and strong sensitivity. We establish the following. (1) If $(X, f)$ is minimal and sensitive, then $(X, f)$ is syndetically sensitive. (2) Weak mixing implies thick sensitivity. (3) If $(X, f)$ is minimal and weakly mixing, then it is thickly syndetically sensitive. (4) If $(X, f)$ is a nonminimal $M$-system, then it is thickly syndetically sensitive. Devaney chaos implies thickly periodic sensitivity. (5) We give a syndetically sensitive system which is not thickly sensitive. (6) We give thickly syndetically sensitive examples but not cofinitely sensitive ones.

\section{Introduction and Preliminaries}

The idea of sensitivity from the work $[1,2]$ by Ruelle and Takens was applied to topological dynamics by Auslander and Yorke in [3] and popularized later by Devaney in [4]. A system $(X, f)$ is called $\varepsilon$-sensitive if there exists a positive $\varepsilon$ (sensitive constant) such that any $x \in X$ is a limit of points $y \in X$ satisfying the condition $d\left(f^{n}(x), f^{n}(y)\right)>\varepsilon$ for some positive integer $n$.

Throughout this paper, a topological dynamical system (TDS for short) $(X, f)$ is a pair where $X$ is a compact metric space without isolated points with metric $d$ and $f: X \rightarrow X$ is a surjective continuous map. For any nonempty subset $Y$ of $X$ and any $r>0$, write $B(Y, r)=\{x \in X: d(x, Y)<r\}$ and $\operatorname{diam}(Y)=\sup \{d(y, z): y, z \in Y\}$. When $Y=\{y\}$ is a singleton, we write $B(y, r)$ for $B(Y, r)$.

Let $S$ be a subset of a positive integer set $\mathbb{Z}_{+}$. $S$ is periodic if there is $T$ such that $\left\{m+k T: k \in \mathbb{Z}_{+}\right\} \subset S$ for some $m \in \mathbb{Z}_{+}$. $S$ is syndetic if it has bounded gaps; that is, there is $N$ such that $\{i, i+1, \ldots, i+N\} \cap S \neq \emptyset$ for every $i \in \mathbb{Z}_{+} . S$ is thick if it contains arbitrarily long runs of positive integers; that is, there is a strictly increasing subsequence $\left\{n_{i}\right\}$ of $\mathbb{N}$ such that $S \supset \cup_{i=1}^{\infty}\left\{n_{i}, n_{i}+1, \ldots, n_{i}+i\right\} . S$ is piecewise syndetic if it is an intersection of a syndetic set with a thick set. $S$ is thickly syndetic if for every $N$ the positions where length $N$ runs begin from a syndetic set. $S$ is thickly periodic if for every $N$ the positions where length $N$ runs begin from a periodic set. Write $N(U, V)=\left\{n \in \mathbb{N}: f^{n}(U) \cap V \neq \emptyset\right\}, N(x, U)=$ $\left\{n \in \mathbb{N}: f^{n}(x) \in U\right\}$. A dynamical system is transitive if for each pair of nonempty open subsets $U, V$ of $X, N(U, V)$ is nonempty. A point $x \in X$ is transitive if the orbit $O(x, f) \equiv\left\{f^{n}(x): n=0,1,2, \ldots\right\}$ is dense in $X$. A system $(X, f)$ is minimal if any $x \in X$ is transitive. We say $(X, f)$ is mixing if for each pair of nonempty open subsets $U, V, N(U, V)$ is cofinite and $(X, f)$ is weakly mixing if $(X \times X, f \times f)$ is transitive. The set $\omega(x, f) \equiv$ $\left\{y\right.$ : there exists an increasing sequence $\left\{n_{i}\right\}$ such that $y=$ $\left.\lim _{i \rightarrow \infty} f^{n_{i}}(x)\right\}$ is said to be the $\omega$-limit set of $x$. We say $x$ is a minimal point (or almost periodic point), if the $\omega$-limit set of $x$ is a minimal set.

Lemma 1. I $(X, f)$ is a transitive nonminimal TDS with $x$ as a transitive point and $S$ as a minimal set of $X$, then $N(x, U(S, \delta))$ is thick for every $\delta>0$.

Proof. For any $k \in \mathbb{Z}_{+}$, since $f$ is uniformly continuous, so $\exists \delta^{\prime}>0$, such that for any $d\left(x_{1}, x_{2}\right)<\delta^{\prime}, d\left(f^{i}\left(x_{1}\right), f^{i}\left(x_{2}\right)\right)<$ $\delta(i=1,2, \ldots, k)$. For any transitive point $x \in X$ and any 
minimal set $S, \exists m \in \mathbb{Z}_{+}$, such that $\left.d\left(f^{m}(x), S\right)\right)<\delta^{\prime}$, so $d\left(f^{m+i}(x), S\right)<\delta(i=1,2, \ldots, k)$; that is, $N(x, U(S, \delta))$ is thick.

Recall that the map $\bar{f}: \mathscr{K}(X) \rightarrow \mathscr{K}(X)$ induced by $f$ on $\mathscr{K}(X)=\{K \subset X: K$ is a nonempty compact subset $\}$ is defined by $\bar{f}(K)=f(K)=\{f(x): x \in K\}, K \in \mathscr{K}(X)$. Then the pair $(\mathscr{K}(X), \bar{f})$ is a dynamical system with the space $\mathscr{K}(X)$ endowed with the Hausdorff distance

$$
\begin{array}{r}
H_{d}\left(K_{1}, K_{2}\right)=\max \left\{\sup \left\{d\left(x_{1}, K_{2}\right): x_{1} \in K_{1}\right\},\right. \\
\left.\sup \left\{d\left(x_{2}, K_{2}\right): x_{2} \in K_{2}\right\}\right\},
\end{array}
$$

$K_{1}, K_{2} \in \mathscr{K}(X)$

Lemma 2 (see [5]). $f$ is weakly mixing $\Leftrightarrow \bar{f}$ is weakly mixing $\Leftrightarrow \bar{f}$ is transitive.

Let $(X, f)$ be a transitive TDS, we say that the system is

(1) a P-system if the periodic points are dense in $X$;

(2) an M-system if the almost periodic points are dense in $X$.

In [6], Glasner and Weiss proved that nonminimal $M$ system is sensitive. And various concepts of stronger sensitivity have been researched recently (see [5-15]).

Lemma 3. If $(X, f)$ is an $M$-system, with $S$ as a minimal subset of $X$ and $V$ as a nonempty open subset of $X$, then $N(V, B(S, \delta))$ is thickly syndetic for any $\delta>0$.

Proof. For any $k \in \mathbb{Z}_{+}$, since $f$ is uniformly continuous, so $\exists \delta^{\prime}>0$, such that for any $d\left(x_{1}, x_{2}\right)<\delta^{\prime}, d\left(f^{i}\left(x_{1}\right), f^{i}\left(x_{2}\right)\right)<$ $\delta(i=1,2, \ldots, k)$. For any transitive point $x \in V$ and any minimal set $S, \exists m \in \mathbb{Z}_{+}$, such that $\left.d\left(f^{m}(x), S\right)\right)<\delta^{\prime} / 2$, so there is a minimal point $x^{\prime} \in V$ with $\left.d\left(f^{m}(x), S\right)\right)<\delta^{\prime}$. Since $x^{\prime}$ is almost periodic, there exists a syndetic set $\left\{n_{i}\right\}$ with $\left.d\left(f^{n_{i}}(x), S\right)\right)<\delta^{\prime}$, so $d\left(f^{n_{i}+j}\left(x^{\prime}\right), S\right)<\delta(j=1,2, \ldots, k, i=$ $1,2,3, \ldots)$. So we have $N(V, B(S, \delta))$ that is thickly syndetic.

\section{Stronger Versions of Sensitivity}

Let $(X, f)$ be a dynamical system. Write $N(U, \epsilon)=\{n \in \mathbb{N}$ : $\left.\operatorname{diam}\left(f^{n}(U)\right)>\epsilon\right\}$. It is easy to see that $(X, f)$ is sensitive if and only if $N(U, \epsilon) \neq \emptyset$ for some $\epsilon>0$ and every nonempty open set $U \subset X$.

\section{Definition 4. A TDS $(X, f)$ is}

(1) syndetically sensitive if $N(U, \epsilon)$ is syndetic for some $\epsilon>0$ and every nonempty open set $U \subset X$,

(2) thickly sensitive if $N(U, \epsilon)$ is thick for some $\epsilon>0$ and every nonempty open set $U \subset X$,

(3) thickly syndetically sensitive if $N(U, \epsilon)$ is thickly syndetic for some $\epsilon>0$ and every nonempty open set $U \subset X$,

(4) thickly periodically sensitive if $N(U, \epsilon)$ is thickly periodic for some $\epsilon>0$ and every nonempty open set $U \subset X$,
(5) cofinitely sensitive if $N(U, \epsilon)$ is cofinite for some $\epsilon>0$ and every nonempty open set $U \subset X$.

From the definition, mixing $\Rightarrow$ cofinite sensitivity $\Rightarrow$ thickly periodically sensitive $\Rightarrow$ thickly syndetical sensitivity $\Rightarrow$ thick sensitivity and syndetical sensitivity.

Theorem 5. If $(X, f)$ is minimal and sensitive, then $(X, f)$ is syndetically sensitive.

Proof. For sensitive constant $\epsilon>0$, by sensitivity of $f$, there exist $x, y \in U$ and $n \in \mathbb{Z}_{+}$such that $d\left(f^{n}(x), f^{n}(y)\right)>\epsilon$ for every nonempty open set $U \subset X$. So there exists an open subset $U_{1} \subset U$ such that $d\left(f^{n}(x), f^{n}\left(U_{1}\right)\right)>\epsilon$. Because $(X, f)$ is minimal, there exists $k \in \mathbb{Z}_{+}$such that $f^{k}(x) \in U_{1} \subset$ $U$, so $d\left(f^{n}(x), f^{k+n}(x)\right) \geq d\left(f^{n}(x), f^{n}\left(U_{1}\right)\right)>\epsilon$. Because $f$ is uniformly continuous, so $\exists \delta \in(0, \epsilon / 4)$ such that for any $d\left(x_{1}, x_{2}\right)<\delta, d\left(f^{i}\left(x_{1}\right), f^{i}\left(x_{2}\right)\right)<\epsilon / 4(i=1,2, \ldots, k)$. Also, $f^{n}(x)$ is a minimal point, so $N\left(f^{n}(x), B\left(f^{n}(x), \delta\right)\right)$ is syndetic. For any $m \in N\left(f^{n}(x), B\left(f^{n}(x), \delta\right)\right)$, we have $d\left(f^{n}(x), f^{m+n}(x)\right)<\delta$, so $d\left(f^{k+n}(x), f^{k+m+n}(x)\right)<\epsilon / 4$; that is,

$$
\begin{aligned}
& d\left(f^{(m+n)}(x), f^{k+m+n}(x)\right) \\
& \geq d\left(f^{n}(x), f^{k+n}(x)\right)-d\left(f^{n}(x), f^{m+n}(x)\right) \\
& \quad-d\left(f^{k+n}(x), f^{k+m+n}(x)\right)>\frac{\epsilon}{2} .
\end{aligned}
$$

This means $N(U, \epsilon / 2)$ is syndetic.

Theorem 6. Weak mixing implies thick sensitivity.

Proof. Let $\epsilon \in(0, \operatorname{diam}(X) / 4)$ and let $V$ be a nonempty open set of $X$. By Lemma 2, $\bar{f}$ is transitive. $X$ is a fixed point of $\bar{f}$ $(\bar{f}(X)=X)$. By density of transitive points of $\bar{f}$ in $\mathscr{K}(X)$, we have $\exists K \subset V$, a transitive point of $\bar{f}$. Because of Lemma 1 , $N\left(K, B_{H_{d}}(X, \epsilon)\right)$ is thick; that is, $N(V, \epsilon)$ is thick (because $N(K, U(X, \epsilon)) \subset N(V, \epsilon))$.

Theorem 7. If $(X, f)$ is a minimal weakly mixing TDS, then it is thickly syndetically sensitive.

Proof. Since $X$ is a fixed point of $(K(X), \bar{f})$, by the proof of Lemma 1 , it is sufficient to prove that $\left\{n: H_{d}\left(f^{n}(V), X\right)<\epsilon\right\}$ is syndetic for any open set $V$ of $X$ and any $\epsilon>0$. Given $V$ and $\epsilon$, let $K \subset V$ be a transitive point for $\bar{f}$. So there exist $n \in \mathbb{Z}_{+}$ such that $H_{d}\left(f^{n}(K), X\right)<\epsilon / 6$. Choose $\gamma>0$ to be an $\epsilon / 6$ modulus of uniform continuity for $f^{n}$ so that $B(K, \gamma) \subset V$. Then $d\left(y_{i}, z_{i}\right)<\gamma$ for $i=1, \ldots, k$ implies $\left\{y_{1}, \ldots, y_{k}\right\} \subset V$ and $H_{d}\left(f^{n}\left(\left\{x, y_{1}, \ldots, y_{k}\right\}\right), f^{n}\left(\left\{x, z_{1}, \ldots, z_{k}\right\}\right)\right)<\epsilon / 6$. Since the orbit of $x$ is dense in $X$, there are strictly increasing integers $\left\{n_{i}: i=1,2, \ldots, k\right\}$ such that $d\left(f^{n_{i}}(x), z_{i}\right)<\gamma$ for $i=1, \ldots, k$. Hence, $H_{d}\left(f^{n}\left(\left\{x, f^{n_{1}}(x), \ldots, f^{n_{k}}(x)\right\}\right), X\right)<\epsilon / 2$. Because $f$ is uniformly continuous, so $\exists \delta \in(0, \epsilon / 4)$ such that for any $d\left(x_{1}, x_{2}\right)<\delta, d\left(f^{i}\left(x_{1}\right), f^{i}\left(x_{2}\right)\right)<\epsilon / 2\left(i=1,2, \ldots, n_{k}\right)$. Since $f^{n}(x)$ is a minimal point, $N\left(f^{n}(x), B\left(f^{n}(x), \delta\right)\right)$ is syndetic. For any $m \in N\left(f^{n}(x), B\left(f^{n}(x), \delta\right)\right.$ ) (i.e., 
$\left.d\left(f^{m+n}(x), f^{n}(x)\right)<\delta\right)$, we have $d\left(f^{m}\left(f^{n+n_{i}}(x)\right), f^{n+n_{i}}(x)\right)=$ $d\left(f^{n_{i}}\left(f^{m+n}(x)\right), f^{n_{i}}\left(f^{n}(x)\right)\right)<\epsilon / 2$, that is,

$$
H_{d}\left(f^{m+n}\left(X_{k}\right), f^{n}\left(X_{k}\right)\right)<\frac{\epsilon}{2} .
$$

Then,

$$
\begin{aligned}
H_{d} & \left(f^{m+n}(V), X\right) \\
& \leq H_{d}\left(f^{m+n}\left(X_{k}\right), X\right) \\
& \leq H_{d}\left(f^{m+n}\left(X_{k}\right), f^{n}\left(X_{k}\right)\right)+H_{d}\left(f^{n}\left(X_{k}\right), X\right)<\epsilon .
\end{aligned}
$$

Theorem 8. If $(X, f)$ is a nonminimal $M$-system, then it is thickly syndetically sensitive.

Proof. Let $A, B$ be minimal sets of $f$ with $d(A, B)=a$ and let $V$ be a nonempty open set of $X$. For any $k \in \mathbb{Z}_{+}$, since $f$ is uniformly continuous, so $\exists \delta>0$, such that for any $d\left(x_{1}, x_{2}\right)<\delta, d\left(f^{i}\left(x_{1}\right), f^{i}\left(x_{2}\right)\right)<a / 4(i=1,2, \ldots, k)$. By Lemma 3, we have $N(V, B(A, \delta)), N(V, B(B, \delta))$, thickly syndetic, so we have $N(V, B(A, \delta)) \cap N(V, B(B, \delta))$, syndetic. Thus, for every $m \in N(V, B(A, \delta)) \cap N(V, B(B, \delta)),\{m, m+$ $1, \ldots, m+k\} \subset N(V, B(A, a / 4)) \cap N(V, B(B, a / 4))$. By arbitrary of $k, N(V, B(A, a / 4)) \cap N(V, B(B, a / 4))$ is thickly syndetic; that is, $N(V, a / 2)$ is thickly syndetic.

Corollary 9. Devaney chaos (or P-system without isolated points) implies thickly periodic sensitivity.

Proof. The proof is similar to Theorem 8 .

\section{Some Examples}

Now we give an example which is syndetically sensitive but not thickly sensitive; especially, $\left(C_{\alpha}, d_{\alpha}\right)$ is not thickly syndetically sensitive.

Example 10. We will use $\mathbb{R} / \mathbb{Z}$ as a model for the circle $S^{1}$. The metric $d^{\prime}$ is defined by $d^{\prime}(a, b)=\min \{|a-b|, 1-|a-b|\}$. Rigid rotation by the real number $\alpha$ is then given by

$$
R_{\alpha}: S^{1} \longrightarrow S^{1}, \quad R_{\alpha}=t+\alpha(\bmod 1) .
$$

Corresponding to the irrational $\alpha$, the Denjoy homeomorphism $d_{\alpha}: S^{1} \rightarrow S^{1}$ is an orientation preserving homeomorphism of the circle characterized by the properties: the rotation number of $d_{\alpha}$ is $\alpha$; there is a Cantor set $C_{\alpha} \subset S^{1}$ on which $d_{\alpha}$ acts minimally; and if $u$ and $v$ are any two components of $S^{1} \backslash C_{\alpha}$ then $d_{\alpha}^{n}(u)=v$ for some integer $n$ (see [4]). There is a Cantor function $h_{\alpha}: S^{1} \rightarrow S^{1}$ that semiconjugates $d_{\alpha}$ with $R_{\alpha}: h_{\alpha}$ being a monotone surjection that collapses the components of $S^{1} \backslash C_{\alpha}$ (and so maps $C_{\alpha}$ onto $S^{1}$ ) with $R_{\alpha} \circ h_{\alpha}=h_{\alpha} \circ d_{\alpha}$.

Let $\left(C_{\alpha}, d_{\alpha}\right)$ be the minimal subsystem of a Denjoy homeomorphism and $c=\max \{\operatorname{diam}(u): u$ is a connected component of $S^{1} \backslash C_{\alpha}$ with $\left.\operatorname{diam}(u)<1 / 4\right\}$. Then $\left(C_{\alpha}, d_{\alpha}\right)$ is syndetically sensitive but not thickly sensitive, and especially, $\left(C_{\alpha}, d_{\alpha}\right)$ is not thickly syndetically sensitive.
Proof. Let $U$ be a nonempty open set of $C_{\alpha}$. For any $x \in U$, there is $y \in U$ such that $h_{\alpha}(x) \neq h_{\alpha}(y)$. Let $\left[h_{\alpha}(x), h_{\alpha}(y)\right]$ be the arc in $S^{1}$ whose endpoints are $x$ and $y$ and whose length is $d^{\prime}\left(h_{\alpha}(x), h_{\alpha}(y)\right)$. Then there exist $w$ and $\delta^{\prime}>0$ such that $w \in B\left(w, \delta^{\prime}\right) \subset\left[h_{\alpha}(x), h_{\alpha}(y)\right]$. Let $u$ be one of the connected components of $S^{1} \backslash C_{\alpha}$ with $\operatorname{diam}(u)=c$ and $p=d_{\alpha}(u) . N\left(w, B\left(p, \delta^{\prime}\right)\right)$ is syndetic. For any $i \in N\left(w, B\left(p, \delta^{\prime}\right)\right), p \in\left[R_{\alpha}^{i}\left(h_{\alpha}(x)\right), R_{\alpha}^{i}\left(h_{\alpha}(y)\right)\right]$. So $\operatorname{diam}\left(d_{\alpha}^{i}(U)\right) \geq d\left(d_{\alpha}^{i}(x),\left(d_{\alpha}^{i}(y)\right)\right)>c$; that is, $\left(C_{\alpha}, d_{\alpha}\right)$ is syndetically sensitive.

On the other hand, we assume that $\left(C_{\alpha}, d_{\alpha}\right)$ is thickly sensitive. Put $k>1 / \epsilon$. Choose an open set $V(\operatorname{diam}(V)$ is little enough) of $C_{\alpha}$ such that $\left\{R_{\alpha}^{i}\left(V^{\prime}\right): i=0,1, \ldots, k\right\}$ are pairwise disjoint where $V^{\prime}=h_{\alpha}(V)$. Since $R_{\alpha}$ is a rotation, $\left\{R_{\alpha}^{m+i}\left(V^{\prime}\right): i=0,1, \ldots, k\right\}$ are pairwise disjoint for every $m \in \mathbb{Z}_{+}$. So $\left\{d_{\alpha}^{m+i}(V): i=0,1, \ldots, k\right\}$ are pairwise disjoint for every $m \in \mathbb{Z}_{+}$. Because of thick transitivity, there exists an integer $n$, such that $\operatorname{diam}\left(d_{\alpha}^{n+i}(V)\right)>\epsilon, i=0,1, \ldots, k$. Since $\left\{d_{\alpha}^{n+i}(V): i=0,1, \ldots, k\right\}$ are pairwise disjoint, so we have the length of $\operatorname{arc} S^{1} 1>\sum_{i=0}^{k} \operatorname{diam}\left(d_{\alpha}^{n+i}(V)>k \epsilon>1\right.$. This is a contradiction.

Example 11. Every uniformly rigid weakly mixing minimal system (see [9], e.g., for the existence of these) is thickly syndetically sensitive but not cofinitely sensitive.

\section{Conflict of Interests}

The authors declare that there is no conflict of interests regarding the publication of this paper.

\section{Acknowledgments}

The authors would like to thank the referees for their helpful remarks. This work is supported by NSFC 11001038 and 11271061, the National High Technology Research and Development Program of China (863 Program) (2012AA01A309), and the Fundamental Research Funds for the Central Universities DC120101112.

\section{References}

[1] D. Ruelle and F. Takens, "On the nature of turbulence," Communications in Mathematical Physics, vol. 20, pp. 167-192, 1971.

[2] D. Ruelle and F. Takens, "Note concerningour paper: "On the nature of turbulence,"' Communications in Mathematical Physics, vol. 23, pp. 343-344, 1971.

[3] J. Auslander and J. A. Yorke, "Interval maps, factors of maps, and chaos," The Tôhoku Mathematical Journal, vol. 32, no. 2, pp. $177-188,1980$.

[4] R. Devaney, Chaotic Dynamical Systems, Addison-Wesley, Reading, Mass, USA, 1989.

[5] G. F. Liao, L. D. Wang, and Y. C. Zhang, "Transitivity, mixing and chaos for a class of set-valued mappings," Science in China A: Mathematics, vol. 49, no. 1, pp. 1-8, 2006.

[6] E. Glasner and B. Weiss, "Sensitive dependence on initial conditions," Nonlinearity, vol. 6, no. 6, pp. 1067-1075, 1993. 
[7] E. Akin and S. Kolyada, "Li-Yorke sensitivity," Nonlinearity, vol. 16, no. 4, pp. 1421-1433, 2003.

[8] H. Wang, X. Long, and H. Fu, "Sensitivity and chaos of semigroup actions," Semigroup Forum, vol. 84, no. 1, pp. 81-90, 2012.

[9] S. Glasner and D. Maon, "Rigidity in topological dynamics," Ergodic Theory and Dynamical Systems, vol. 9, no. 2, pp. 309320, 1989.

[10] W. Huang and X. Ye, "An explicit scattering, non-weakly mixing example and weak disjointness," Nonlinearity, vol. 15, no. 3, pp. 849-862, 2002.

[11] T. K. S. Moothathu, "Stronger forms of sensitivity for dynamical systems," Nonlinearity, vol. 20, no. 9, pp. 2115-2126, 2007.

[12] H. Y. Wang, J. C. Xiong, and F. Tan, "Furstenberg families and sensitivity," Discrete Dynamics in Nature and Society, vol. 2010, Article ID 649348, 12 pages, 2010.

[13] H. Liu, E. Shi, and G. Liao, "Sensitivity of set-valued discrete systems," Nonlinear Analysis: Theory, Methods \& Applications, vol. 71, no. 12, pp. 6122-6125, 2009.

[14] P. Sharma and A. Nagar, "Inducing sensitivity on hyperspaces," Topology and its Applications, vol. 157, no. 13, pp. 2052-2058, 2010.

[15] X. Ye and R. Zhang, "On sensitive sets in topological dynamics," Nonlinearity, vol. 21, no. 7, pp. 1601-1620, 2008. 


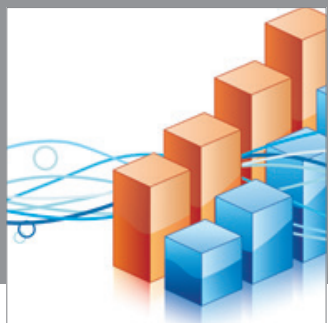

Advances in

Operations Research

mansans

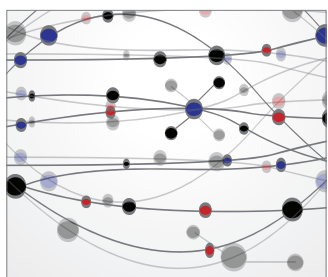

The Scientific World Journal
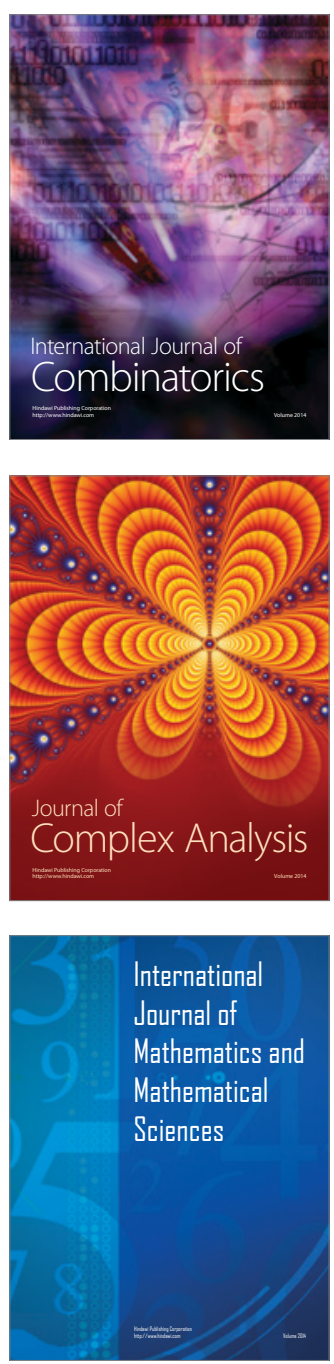
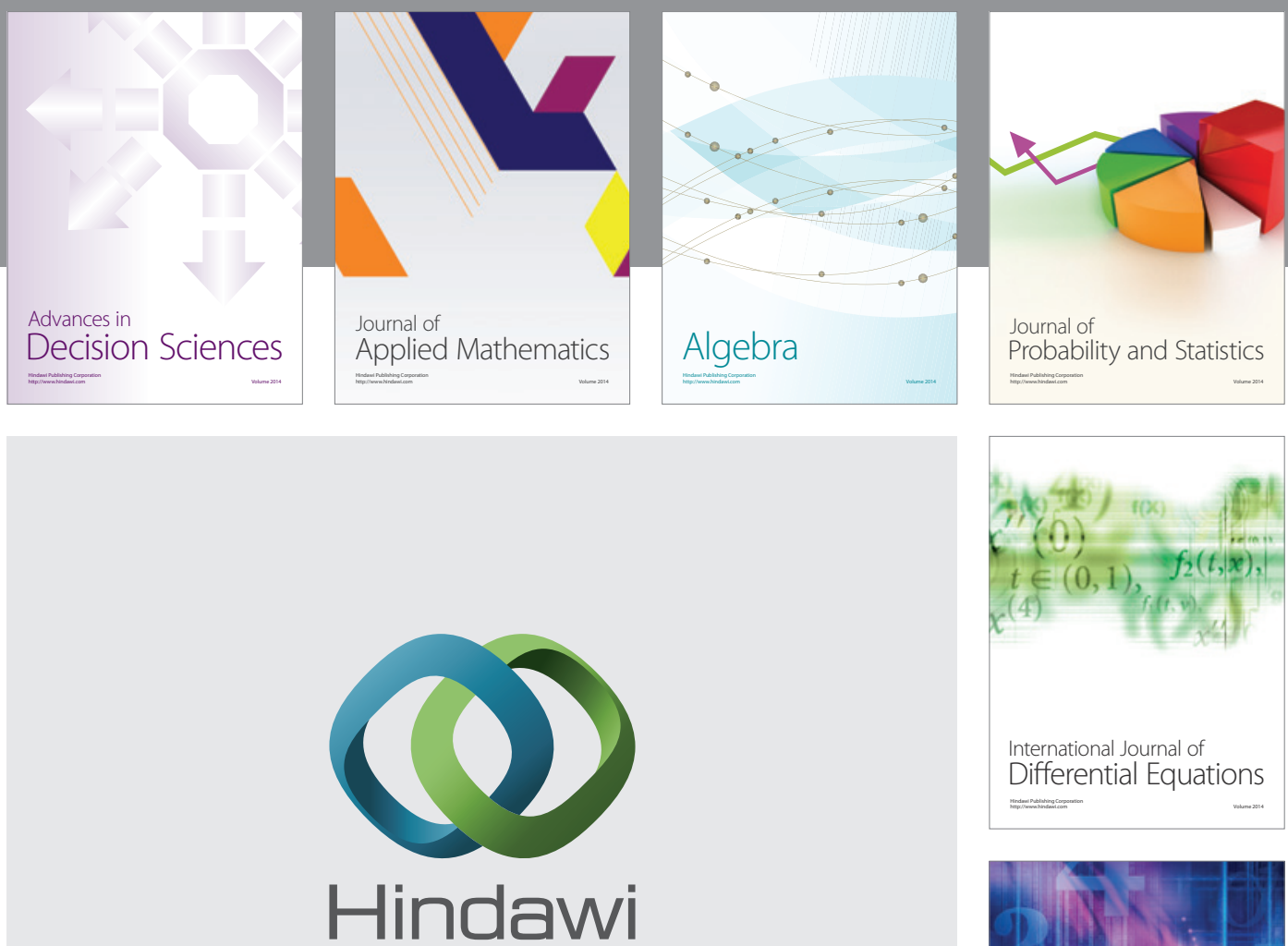

Submit your manuscripts at http://www.hindawi.com
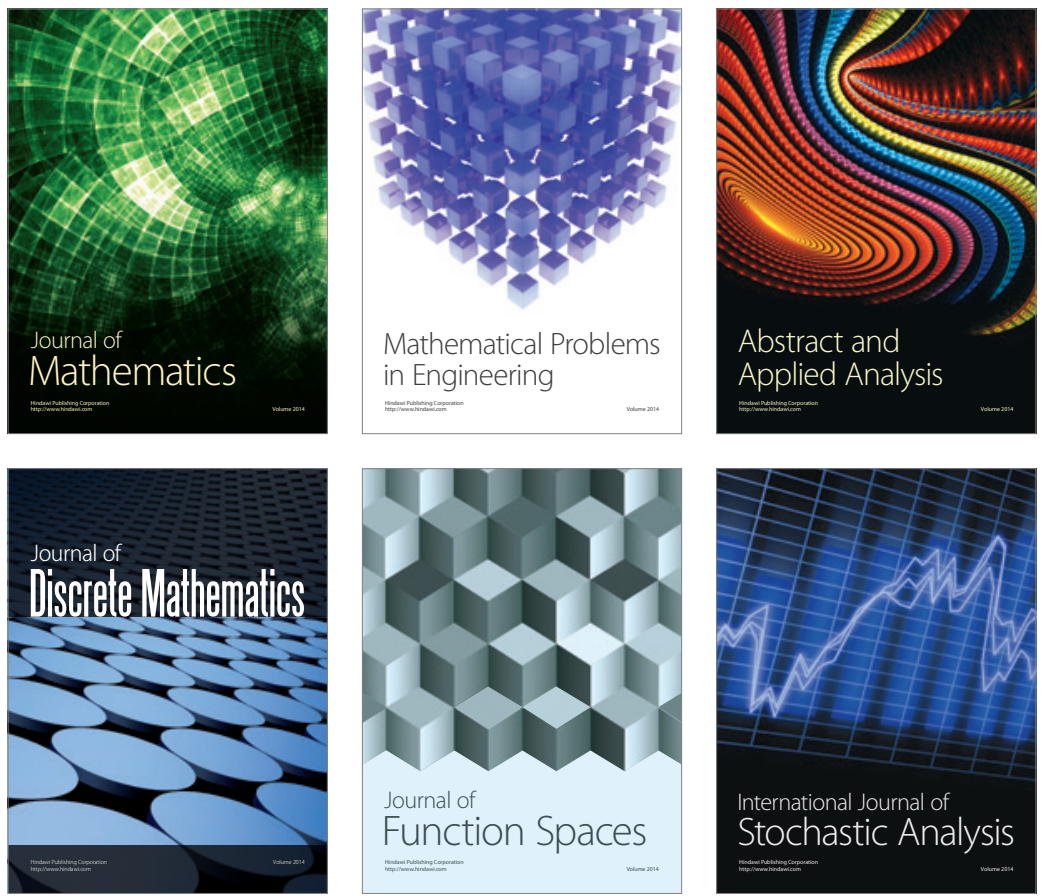

Journal of

Function Spaces

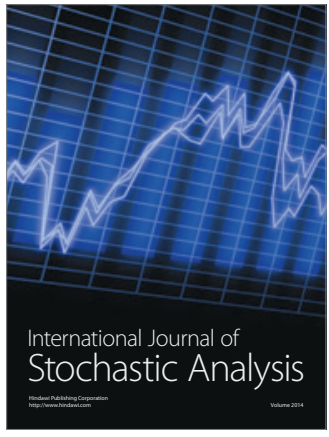

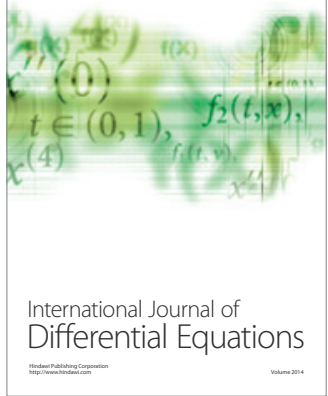
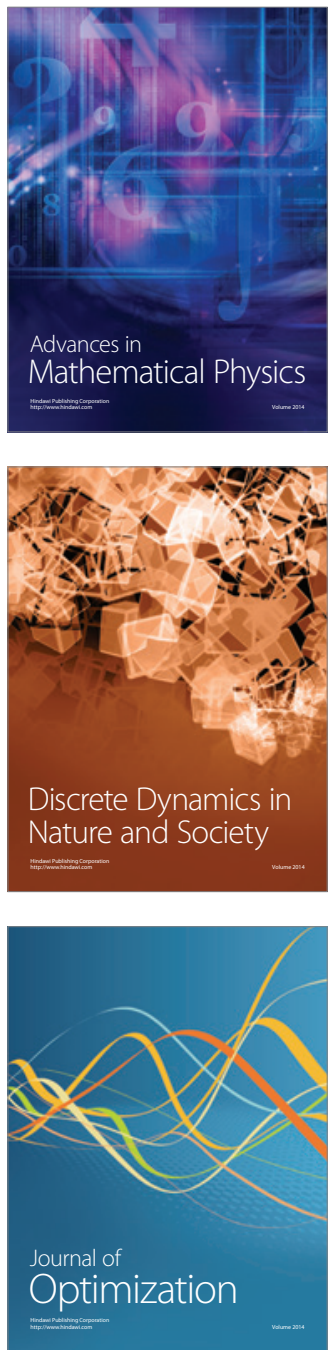